\title{
Determinantes intrapessoais e interpessoais que norteiam as recaídas na dependência química
}

\author{
Intrapersonal and interpersonal determinants that guide relapses in chemical dependency \\ Determinantes intrapersonales e interpersonales que guían las recaídas en la \\ dependencia química
}

Julyana de Souza Araujo ${ }^{1 *}$, Jéssica Moreno Cordeiro ${ }^{1}$, Heloisa Marilak Alves Veloso ${ }^{1}$, Júlia Romana de Santana Costa ${ }^{1}$, José Lopes Pereira Júnior ${ }^{1}$.

\section{RESUMO}

Objetivo: Descrever as causas intrapessoais e interpessoais de recaídas dos dependentes químicos. Métodos: Trata-se de um estudo por revisão integrativa por busca nas bases de dados: PubMed, Scientific Eletronic Library Online (SciELO), Literatura Latino-Americana e do Caribe em Ciências da Saúde (LILACS) e Cochrane Libary. Os descritores: "drugs" OR "substance" OR "abuse drugs" OR "addiction" AND "relapse". Aplicado análise temática de conteúdo, identificado por meio da leitura integral, temas predominantes entre os objetivos, o método, a abordagem ou a técnica estudada e a síntese dos resultados. Resultados: Incluídos 17 artigos de 2003 a 2019. Amostras de 12 a 608 indivíduos com análises de entrevistas em grupo e focais, semiestruturadas, fenomenológicas questionários e auto relato. Os estudos apresentaram como as poli drogas prediz o aumento das recaídas; a abstinência de metanfetamina mais à falta de atenção; a relação do tempo de abuso da substância com a maior chance de recaída; como dependentes químicos criminosos são tratados; como os sonhos geram sensações de relevante interpretação; e o apoio emocional e físico como fatores de proteção. Considerações Finais: A influência do meio para a recaída, onde as ordens físicas, psíquicas, emocionais, sociais e profissionais são determinantes para a recuperação.

Palavras-chave: Drogas, Substância, Recaída.

\begin{abstract}
Objective: Describe the intrapersonal and interpersonal causes of relapses of drug addicts. Methods: This is a study by integrative review by searching in the databases: PubMed, Scientific Eletronic Library Online (SciELO), Literatura Latino-Americana e do Caribe em Ciências da Saúde (LILACS) and Cochrane Libary. The descriptors: "drugs" OR "substance" OR "abuse drugs" OR "addiction" AND "relapse". Applied thematic content analysis, identified through full reading, predominant themes among the objectives, method, approach or technique studied and the synthesis of results. Results: 17 articles from 2003 to 2019 were included. Samples from 12 to 608 individuals with analysis of group and focal interviews, semi-structured, phenomenological questionnaires and self-report. Studies have shown how polydrugs predict increased relapse; methamphetamine withdrawal plus inattention; the relationship between the time of substance abuse and the highest chance of relapse; how criminal drug addicts are treated; how dreams generate sensations of relevant interpretation; and emotional and physical support as protective factors. Final Considerations: The influence of the environment for relapse is concluded, where the physical, psychological, emotional, social and professional orders are decisive for recovery.
\end{abstract}

Key words: Drugs, Substance, Relapse.

${ }^{1}$ Faculdade de Ciências Humanas, Exatas e da Saúde do Piauí (FAHESP), Parnaíba - PI.

*E-mail: julyana.souza.a@gmail.com

SUBMETIDO EM: 11/2020

ACEITO EM: 1/2021

PUBLICADO EM: 3/2021 


\section{RESUMEN}

Objetivo: Describir las causas intrapersonales e interpesonales de las recaídas de los adictos a las drogas. Métodos: Se trata de un estudio de revisión integradora mediante la búsqueda en las bases de datos: PubMed, Scientific Eletronic Library Online (SciELO), Literatura Latino-Americana e do Caribe em Ciências da Saúde (LILACS) and Cochrane Libary. Los descriptores: "drogas" O "sustancia" O "drogas de abuso" O "adicción" Y "recaída". Análisis de contenido temático aplicado, identificado mediante lectura completa, temas predominantes entre los objetivos, método, enfoque o técnica estudiada y síntesis de resultados. Resultados: Se incluyeron 17 artículos de 2003 a 2019. Muestras de 12 a 608 individuos con análisis de entrevistas grupales y focales, cuestionarios semiestructurados, fenomenológicos y autoinforme. Los estudios han demostrado cómo los polidrogas predicen un aumento de las recaídas; abstinencia de metanfetamina más falta de atención; la relación entre el momento del abuso de sustancias y la mayor probabilidad de recaída; cómo se trata a los drogadictos criminales; cómo los sueños generan sensaciones de interpretación relevante; y apoyo emocional y físico como factores protectores. Consideraciones Finales: La influencia del entorno para la recaída, donde los órdenes físico, psicológico, emocional, social y profesional son determinantes para la recuperación.

Palabras clave: Drogas, Sustancia, Recaída.

\section{INTRODUÇÃO}

O aumento do consumo de drogas está diretamente associado ao prazer contemporâneo, assim como a cultura do consumo, além de possuir relação com a situação de vulnerabilidade na qual vive uma significativa parcela da sociedade, destacando as classes mais desfavoráveis. Pois, é de conhecimento comum que grandes parcelas da sociedade vivem diariamente à mercê de ameaças relacionadas às suas condições de vidas precárias da mesma maneira pela própria exclusão social (ALMEIDA RBF, 2018).

A dependência de drogas é um transtorno mental complicado, porque vários níveis de sistemas biológicos e psíquicos são recrutados para seu desenvolvimento, dentre os quais o sistema de recompensa e antirecompensa contribuem sinergicamente para a recaída após um período de retirada prolongada (LIL $L$ e HSIAO SC, 2018).

O vício é um transtorno recorrente crônico, em que a maioria dos indivíduos viciados que optam por parar o consumo de drogas não conseguem suportar abstinência a longo prazo. Apesar de décadas de pesquisa, a alta prevalência de recaída no uso de substâncias de abuso continua a desafiar o campo. $O$ abuso e a dependência de drogas têm profundos custos sociais e econômicos em todo o mundo (LU L, et al., 2009).

Entende-se por recaída o insucesso ao tentar alcançar um objetivo estabelecido como meta de um indivíduo por um período determinado, ou ainda a relação das tentativas de parar ou diminuir o consumo de substâncias alucinógena ou entorpecente. É definido ainda como a recidiva ao uso do álcool ou drogas, com a mesma relação de consumo que a pessoa tinha antes de iniciar um programa de tratamento ou recuperação (ÁLVARES AMA, 2007).

A recaída é especialmente provável quando os indivíduos em recuperação encontram fatores de risco como estresse, solidão, ausência de perspectivas futuras ou ainda acesso à locais associados às drogas (FARRELL MR, et al., 2018). Soma-se a isso fatores pessoais, espirituais, financeiros e estrutura familiar, além de outros.

Portanto, é possível alterar os padrões de vício e as principais fontes que levam o indivíduo as recaídas, sendo então aplicado intervenções desenvolvimentais e ambientais antes mesmo das ações do dependente, como ocorre na psicoterapia. O tratamento da toxicodependência tem diversas limitações tais como a dessemelhança dos dependentes, os diversos tipos e classes das substâncias no mercado legal e ilegal, os custos econômicos privados e governamentais, as dificuldades de gerir os recursos humanos e as dificuldades de materiais especializados (RIGGOTO SD e GOMES WB, 2002). 
Apesar de existirem uma gama de fatores de risco e proteção e que, para cada sujeito ou grupos, eles se farão ou não presentes, compreender quais fatores estão ou podem estar implicados no uso de drogas pode estimular o incremento de estratégias de intervenção que sejam mais eficazes na promoção de saúde.

Diante disso, o presente artigo apresenta uma revisão da integrativa literatura científica com o objetivo de descrever as causas intrapessoais e interpessoais de recaídas dos dependentes químicos. Além disso, uma visão geral das principais causas que levam a recaídas, assim como a importância do suporte da equipe de saúde multiprofissional e o vínculo familiar.

\section{MÉTODOS}

Foi realizada uma revisão integrativa por meio de uma busca na literatura internacional e nacional, indexada nas seguintes bases de dados: Pubmed, SciELO, Lilacs e Cochrane Library. Os descritores utilizados foram: "drugs" OR "substance" OR "abuse drugs" OR "addiction" AND "relapse". A busca incluiu artigos publicados de 2003 a 2020. A consulta nas bases de dados foi realizada no mês de agosto de 2020, a partir de uma leitura dos resumos capturados.

Após um primeiro levantamento, os artigos pré-selecionados e aqueles sobre os quais pairassem dúvidas quanto à inclusão foram submetidos à leitura na íntegra, como nova rodada de decisões quanto à sua inclusão no estudo. Casos dúbios foram resolvidos por avaliação conjunta dos autores, os quais não tiveram contato entre si durante a seleção.

Foram selecionados e incluídos na revisão estudos que elencassem fatores/situações responsáveis pela recaída de dependentes químicos no uso de drogas. Não foram selecionados estudos que mencionassem apenas o tratamento desses usuários. Os artigos que se referiam somente aos fatores que levavam os indivíduos a "entrarem" no uso de drogas também não foram considerados. Após leitura de cada um dos artigos selecionados, em sua versão completa, foi empregada análise temática de conteúdo dos artigos, identificando, por meio da leitura integral de cada estudo, temas predominantes na ligação entre os objetivos, o método, a abordagem ou a técnica estudada e a síntese dos resultados apresentados.

O fluxograma de busca e seleção, com a distribuição dos artigos encontrados nas diferentes condições estabelecidas como critérios de inclusão e exclusão (Figura 1).

Figura 1 - Diagrama de fluxo da avaliação e seleção do artigo.

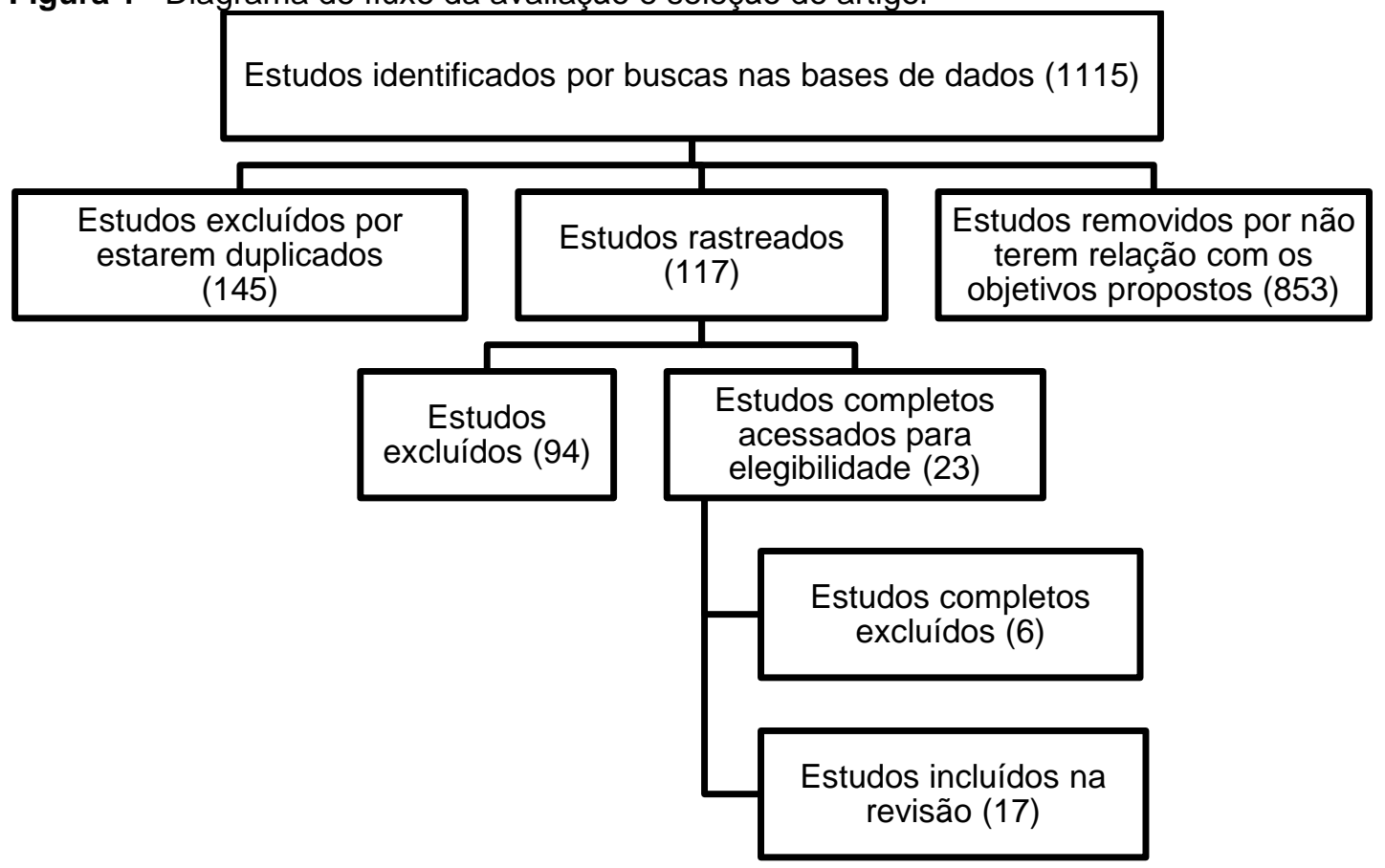

Fonte: Araujo JS, et al., 2020. 


\section{RESULTADOS}

Inicialmente, identificou-se 1115 estudos por buscas nas bases de dados, dentre esses houve exclusão de 145 por estarem duplicados, e mais 853 por não terem relação com o objetivo proposto. Aplicando-se demais critérios de elegibilidade foram excluídos mais 94 estudos, sendo incluídos 29 estudos completos para elegibilidade, desses 6, após leitura e avaliação criteriosa foram excluídos, restando 17 estudos para serem incluídos nessa revisão. Os estudos datam de 2003 a 2019, sendo os mesmos feitos em vários países, oito foram feitos no Brasil (Quadro 1).

Os estudos trabalharam com amostras variáveis, de 12 a 608 indivíduos e envolveram instrumentos de análises a partir de entrevistas, sendo uma em grupo e focais, outras semiestruturadas, uma fenomenológica, além disso, foram utilizados também questionários, e um autorrelato. Os estudos se dividiram em desenhos de pesquisa qualitativos e quantitativos, envolvendo coorte, transversal, prospectivos, além de ensaio randomizado.

Os desfechos dos estudos retratam os fatores que predizem os pacientes a apresentarem recaídas durante o tratamento contra dependência de drogas, um dos estudos relata que o consumo de várias drogas pode predizer o aumento dessas recaídas (CHEN T, et al. 2018).

No artigo de Chen Y, et al. (2015) há posicionamento acerca de que a abstinência do uso de metanfetamina correlacionada à falta de atenção predispõem as recaídas, Lopes-Rosa R, et al. (2015) estabelece que há maior chance de retorno associado ao tempo de abuso da substância, quanto maior o tempo de abuso, as chances de o paciente apresentar recaídas também tendem a aumentar, no estudo de Xiong $\mathrm{H}$ e Jia J (2019) leva em consideração o quesito de como os dependentes químicos são tratados durante o tratamento, no caso de criminosos não serem abordados como tal, e sim como pacientes que precisam de ajuda para sair da situação das drogas e não reincidirem no abuso, bem como quanto maior o apoio social a esses exusuários durante a reabilitação melhor será a forma como os pacientes irá responder ao tratamento.

Há relato no estudo de Da Silva TR e Nappo AS (2019) sobre sonhos como desencadeadores de sensações, como fazendo o paciente reviver a sensação do consumo, favorecendo assim que haja uma possível recaída, ademais a forma como os pacientes interpretam seus sonhos podem subsidiar possíveis intervenções que favoreçam a terapêutica.

Outros cinco trazem como desfechos que o apoio emocional e físico para esses pacientes são fatores protetivos, motivos pelos quais protegem os ex-usuários a não retornarem ao abuso de drogas, além disso, alegam que fatores que predispõe as recaídas são os fatores sociais, como a pobreza, fatores socioculturais e atrelado a isso, a própria falta de apoio familiar (CHAN GHY, et al., 2019; YANG M, et al. 2015; BATOOL S, et al., 2017; CARVALHO FRM, et al., 2011).

Contatou-se que mulheres têm mais chances recaídas que homens, pois vivenciam as emoções negativas como fator predisponente (OVESI S, et al., 2020). O artigo de Buriola AA, et al. (2011) aponta que os fatores relacionados à recaída são fatores interpessoais e intrapessoais vivenciados pelos ex usuários. Ainda podese citar que outros dois estudos trazem fatores como frustrações, inatividade, perdas, comorbidades, desemprego, impotência como sentimentos que podem levar as recaídas (CARVALHO FRM, et al., 2011; BUCHELE F, et al., 2004).

Pode-se argumentar que o desfecho do estudo de Büchele $F$, et al. (2004) mostra que o ambiente frequentado por quem deseja abandonar o vício em drogas pode influenciar diretamente na sua recuperação. No artigo de Carvalho FRM, et al. (2011), revisa a necessidade de aprimorar os processos de prevenção às recaídas, por meio de melhorias nos serviços prestados, principalmente pelo governo. Ademais, no estudo de Soccol KLS, et al. (2019), mostra que as recaídas estão atreladas, principalmente as violências, fragilizações dos vínculos afetivos e perdas familiares, e que há prejuízos sociais e na saúde das pacientes estudadas. 
Quadro 1 - Principais desfechos encontrados nos estudos incluídos na revisão.

\begin{tabular}{|c|c|c|c|c|c|}
\hline $\begin{array}{l}\text { Local de } \\
\text { publicação }\end{array}$ & $\begin{array}{l}\text { Primeiro autor e ano } \\
\text { de publicação }\end{array}$ & População & Tipo de Estudo & Instrumento de Análise & Principais Desfechos \\
\hline China & CHEN T, et al. (2018) & $\mathrm{N}: 503$ & $\begin{array}{l}\text { Qualitativo } \\
\text { Coorte }\end{array}$ & Entrevista & $\begin{array}{l}\text { Os padrões de poli consumo diferem entre os pacientes } \\
\text { dependentes de heroína. Uma história de poli consumo de } \\
\text { drogas pode predizer o risco de recaída de heroína entre os } \\
\text { pacientes nesses programas de reabilitação obrigatória. }\end{array}$ \\
\hline China & CHEN Y, et al. (2015) & $\mathrm{N}: 42$ & $\begin{array}{l}\text { Qualitativo e } \\
\text { Quantitativo } \\
\text { Prospectivo }\end{array}$ & $\begin{array}{c}\text { Entrevista } \\
\text { Teste de Desempenho Contínuo de Conners (CPT) } \\
\text { Wisconsin Teste de classificação de cartas (WCST) } \\
\text { lowa Gambling Task (IGT) } \\
\text { Escala de Impulsividade Barratt (BIS) }\end{array}$ & $\begin{array}{l}\text { Uma curta duração da abstinência de metanfetamina no } \\
\text { início do estudo atenção insuficiente foram fatores de risco } \\
\text { significativos de recaída entre os usuários de de } \\
\text { metanfetamina em um programa de tratamento de } 12 \\
\text { semanas. }\end{array}$ \\
\hline Brasil & $\begin{array}{l}\text { LOPES-ROSA R, et al. } \\
(2017)\end{array}$ & $\mathrm{N}: 89$ & $\begin{array}{l}\text { Qualitativo e } \\
\text { Quantitativo } \\
\quad \text { Coorte }\end{array}$ & $\begin{array}{c}\text { Autorrelato } \\
\text { Índice de Gravidade do Vício em Adolescentes } \\
\text { (T-ASI) } \\
\text { Cronograma para Transtornos Afetivos e } \\
\text { Esquizofrenia para Crianças em Idade Escolar } \\
\text { (K-SADS-PL) }\end{array}$ & $\begin{array}{l}\text { Associações estatisticamente significativas foram } \\
\text { observadas entre recaída no primeiro mês e tempo de uso } \\
\text { de cocaína / crack e tempo de internação. }\end{array}$ \\
\hline China & XIONG H, JIA J. (2018) & $\mathrm{N}: 608$ & $\begin{array}{l}\text { Qualitativo e } \\
\text { Quantitativo } \\
\text { Prospectivo }\end{array}$ & Questionário & $\begin{array}{l}\text { O apoio social sobre a situação dos ex-usuários de drogas } \\
\text { durante o período da reabilitação pode fornecer um suporte } \\
\text { para melhorias no tratamento. } \\
\text { Quando esses pacientes em reabilitação são tratados de } \\
\text { uma melhor forma, sendo inserido como pacientes e não } \\
\text { como criminosos, há assim uma melhor adesão ao } \\
\text { tratamento. }\end{array}$ \\
\hline Brasil & $\begin{array}{l}\text { DA SILVA TR, NAPPO } \\
\text { SA. (2019) }\end{array}$ & $\mathrm{N}: 21$ & $\begin{array}{l}\text { Qualitativa } \\
\text { Prospectivo }\end{array}$ & Entrevista & $\begin{array}{l}\text { Os sonhos relacionados ao crack obrigam o indivíduo, de } \\
\text { forma involuntária e incontrolável, reviver a cultura do uso, } \\
\text { ou seja, o consumo, os ambientes, os colegas de uso, o } \\
\text { gosto, os cheiros e o prazer. }\end{array}$ \\
\hline China & $\begin{array}{l}\text { CHAN GHY, et al. } \\
(2019)\end{array}$ & $\mathrm{N}: 103$ & $\begin{array}{l}\text { Qualitativo } \\
\text { Prospectivo }\end{array}$ & Entrevistas individuais e grupos focais & $\begin{array}{l}\text { Resultados mostram que a necessidade psicológica de } \\
\text { relacionamento é um determinante importante de se } \\
\text { retornar as drogas ou não, por meio dos mecanismos de } \\
\text { interacãã existentes nas dimensões de afiliacãa e e } \\
\text { intimidade. A implicação é que outras pessoas significativas } \\
\text { podem desenvolver incondicionalmente carinho e empatia } \\
\text { nas relaçóes de apoio com usuários de drogas, de modo a } \\
\text { melhorar a satisfação das necessidades psicológicas e } \\
\text { reduzir o risco de recaída nas drogas. }\end{array}$ \\
\hline
\end{tabular}




\begin{tabular}{|c|c|c|c|c|c|}
\hline $\begin{array}{l}\text { Local de } \\
\text { publicação }\end{array}$ & $\begin{array}{l}\text { Primeiro autor e ano } \\
\text { de publicação }\end{array}$ & População & Tipo de Estudo & Instrumento de Análise & Principais Desfechos \\
\hline China & $\begin{array}{l}\text { LIU L, HSIAO SC. } \\
\underset{(2013)}{ }\end{array}$ & $\mathrm{N}: 46$ & $\begin{array}{l}\text { Qualitativa } \\
\text { Prospectivo }\end{array}$ & Entrevistas & $\begin{array}{l}\text { Exercícios de tipo militar, cronograma apertado, não } \\
\text { garantem a manutenção da abstinência após liberação. } \\
\text { Tratamento em conjunto da sociedade e da família, dando } \\
\text { um suporte emocional, mental, social e familiar aos } \\
\text { usuários, acompanhamento esse que deve ser trabalhado } \\
\text { de maneira longitudinal. }\end{array}$ \\
\hline China & YANG M, et al. (2015) & $N: 20$ & $\begin{array}{l}\text { Qualitativa } \\
\text { Prospectivo }\end{array}$ & Entrevistas & $\begin{array}{l}\text { As tribulações durante a tentativa de abstinência possuem } \\
\text { o fator agravante principal para a recidiva, sendo elas as } \\
\text { condições socioeconômicas de pobreza, a falta de apoio } \\
\text { familiar e suporte social, principalmente quando se refere a } \\
\text { discriminação e o cadastro da carteira de identidade no } \\
\text { sistema policial, são formas de exclusão da sociedade } \\
\text { tornando mais difícil permanecer em abstinência. }\end{array}$ \\
\hline Paquistão & $\begin{array}{l}\text { BATOOL S, et al. } \\
(2017)\end{array}$ & N: 119 & $\begin{array}{l}\text { Quantitativo } \\
\text { Qualitativo } \\
\text { Transversal }\end{array}$ & Questionário e entrevistas & $\begin{array}{l}\text { A cronicidade do uso de drogas foi detectada como um dos } \\
\text { fatores agravantes para a permanência no estilo de vida } \\
\text { tóxico, além das questões socioculturais, econômicas e } \\
\text { familiares. }\end{array}$ \\
\hline Irã & OVEISI S, et al. (2017) & $N: 60$ & $\begin{array}{l}\text { Quantitativo } \\
\text { Qualitativo } \\
\text { Ensaio clínico } \\
\text { randomizado }\end{array}$ & Entrevista & $\begin{array}{l}\text { Os resultados mostraram que a entrevista motivacional } \\
\text { pode diminuir o desejo de usar e a probabilidade de uso } \\
\text { entre usuários de drogas. }\end{array}$ \\
\hline Costa Rica & $\begin{array}{c}\text { ÁLVARES TB, } \\
\text { MORALES SJ. (2014) }\end{array}$ & $\mathrm{N}: 107$ & $\begin{array}{l}\text { Quantitativo } \\
\text { Qualitativo } \\
\text { Transversal }\end{array}$ & Entrevista & $\begin{array}{l}\text { Constatou-se no presente estudo que as mulheres } \\
\text { percebem os estados emocionais negativos como situações } \\
\text { de maior risco de recaída do que os homens. Ou seja, o } \\
\text { sexo e a forma de lidar com os sentimentos, estão } \\
\text { interligados as recaídas. }\end{array}$ \\
\hline Brasil & $\begin{array}{l}\text { CARVALHO FRM, et al. } \\
(2010)\end{array}$ & $\mathrm{N}: 12$ & $\begin{array}{l}\text { Qualitativo } \\
\text { Prospectivo }\end{array}$ & Entrevista & $\begin{array}{l}\text { O meio influencia a recaída; o não reconhecimento da } \\
\text { impotência perante o vício; dificuldade de lidar com } \\
\text { frustrações; a inatividade desperta o desejo pelo uso de } \\
\text { substâncias psicoativas; perdas, comorbidades e o } \\
\text { reconhecimento da impotência motivam a busca de } \\
\text { tratamento. }\end{array}$ \\
\hline Brasil & $\begin{array}{l}\text { BURIOLA AA, et al. } \\
\text { (2015) }\end{array}$ & $\mathrm{N}: 15$ & $\begin{array}{l}\text { Qualitativo } \\
\text { Prospectivo }\end{array}$ & Entrevista semiestruturada & $\begin{array}{l}\text { Percebeu-se que os principais motivos que levam o } \\
\text { indivíduo dependente químico a reiniciar o uso das } \\
\text { substâncias psicoativas emergiram em duas categorias, } \\
\text { sendo estas os determinantes interpessoais e intrapessoais. }\end{array}$ \\
\hline
\end{tabular}




\begin{tabular}{|c|c|c|c|c|c|}
\hline $\begin{array}{l}\text { Local de } \\
\text { publicação }\end{array}$ & $\begin{array}{l}\text { Primeiro autor e ano } \\
\text { de publicação }\end{array}$ & População & Tipo de Estudo & Instrumento de Análise & Principais Desfechos \\
\hline Brasil & $\begin{array}{l}\text { DA SILVA ML, et al. } \\
\qquad(2013)\end{array}$ & $\mathrm{N}: 50$ & $\begin{array}{l}\text { Quantitativo } \\
\text { Prospectivo }\end{array}$ & Questionário semiestruturado & $\begin{array}{l}\text { É importante identificar os fatores de risco referentes às } \\
\text { recaídas, principalmente os ligados a conflitos familiares e } \\
\text { inabilidade de lidar com sentimentos negativos, e fatores } \\
\text { sociais que podem vir a influenciar como festas, situacãa de } \\
\text { rua e locais que propiciam o uso de substâncias. Ademais, } \\
\text { pode-se citar também como fatores que propiciam a a } \\
\text { recáda, convivio com pessoas que também façam uso, a } \\
\text { própria rotina do paciente. Como fatores de proteção pode- } \\
\text { se citar a religiâa, grupos de apoio, além disso, foi citado o } \\
\text { apoio profissional. }\end{array}$ \\
\hline Brasil & $\begin{array}{l}\text { BÜCHELE F, et al. } \\
(2003)\end{array}$ & $\mathrm{N}: 25$ & $\begin{array}{l}\text { Quantitativo } \\
\text { Qualitativo } \\
\text { Prospectivo }\end{array}$ & Questionário & $\begin{array}{l}\text { Algumas situaçães sociais como festas e jantares, podem } \\
\text { exercer influência no que se refere ao uso da droga. Outros } \\
\text { problemas como o desemprego, a falta de um lugar para } \\
\text { morar também abalam o dependente em processo de de } \\
\text { recuperação e se suas respostas de enfrentamento a essas } \\
\text { dificuldades não forem positivas certamente ele enfrentará } \\
\text { o risco de recidiva. Estas situaçóes de ordem física, psíquica } \\
\text { e social, podem influenciar tanto no sucesso quanto no } \\
\text { fracasso de uma recuperação. }\end{array}$ \\
\hline Brasil & $\begin{array}{l}\text { CARVALHO FRM, et al. } \\
(2010)\end{array}$ & $\mathrm{N}: 12$ & $\begin{array}{l}\text { Qualitativo } \\
\text { Prospectivo }\end{array}$ & Entrevista semiestruturada & $\begin{array}{l}\text { Deve-se revisar a necessidade de aprimoramento nos } \\
\text { processos de prevenção às recaídas, garantindo um maior } \\
\text { apoio governamental, que incentive os tratamentos } \\
\text { ofertados, melhorando principalmente estruturas } \\
\text { hospitalares e extra-hospitalares. Juntando a isso, é } \\
\text { necessário o favorecimento da uniâo de fatores físicos, } \\
\text { emocionais, psíquicos e sociais, a fim de prestar um apoio } \\
\text { completo, para desfavorecer as recaídas. }\end{array}$ \\
\hline Brasil & $\begin{array}{c}\text { SOCCOL KLS, et al. } \\
\text { (2017) }\end{array}$ & $\mathrm{N}: 20$ & $\begin{array}{l}\text { Qualitativo } \\
\text { Prospectivo }\end{array}$ & Entrevista fenomenológica & $\begin{array}{l}\text { Mostrou-se que as recaídas ocorrem com a finalidade de } \\
\text { esquecimento de problemas, ao buscar no retorno às } \\
\text { drogas, bem-estar. A recaída vivida por mulheres que } \\
\text { sofreram violências, fragilização dos vínculos afetivos ou } \\
\text { perdas familiares. Ao conhecer as histórias atreladas às } \\
\text { mulheres dos estudos, foi possível reconhecer o que as } \\
\text { levou a recaída. Além de prejuízos a saúde, pode vir a terem } \\
\text { problemas sociais também. }\end{array}$ \\
\hline
\end{tabular}

Fonte: Araujo JS, et al., 2020. 


\section{DISCUSSÃO}

O estudo de Carvalho FRM, et al. (2011) determinou a influência do meio para o episódio de recaída. Além disso, outros dois estudos determinaram que situações de ordem física, psíquica, emocional e social, podem influenciar tanto no sucesso quanto no fracasso de uma recuperação (BÜCHELE F, et al., 2004; CARVALHO FRM, et al., 2011).

Sendo assim, o ideal, para uma baixa na recidiva das drogas, é o tratamento em conjunto, da sociedade e da família, dando um suporte emocional, mental, social e familiar aos usuários de drogas. Esse acompanhamento deve ser trabalhado e executado de forma longitudinal, por profissionais da área da saúde, que procurem qualificar seus conhecimentos acerca deste tema, com a finalidade de estarem aptos a prestar um cuidado integral a esse grupo (LIL L e HSIAO SC, 2018; CARVALHO FRM, et al., 2011).

Partindo da análise da recaída como sobre influência de fatores múltiplos, percebeu-se, ainda, que os principais motivos que levam o indivíduo dependente químico a reiniciar o uso das substâncias psicoativas emergiram em duas categorias, sendo estas os determinantes interpessoais e intrapessoais (BURIOLA AA, et al., 2011).

Primeiramente, deve-se destacar que a recaída corresponde a uma falha na tentativa de parar ou de reduzir o consumo de psicoativos. Ou seja, pode-se entender, também, como a falha ao tentar atingir esses objetivos após um período de abstinência (VARSTERS GP e PILLON SC, 2011).

Dessa forma, os determinantes interpessoais são influências marcadas pelo contexto no qual o indivíduo está inserido, seja em seu âmbito familiar, afetivo ou social. Ter a capacidade de identificar esses fatores de risco, e compreender sua inabilidade em lidar com os mesmos, poderá melhorar suas habilidades e estratégias no enfrentamento e/ou antecipação de comportamentos de recaída (FERNANDES SS, et al., 2017).

Nessa conjuntura, os determinantes intrapessoais são aqueles que se relacionam com as expectativas de resultados individuais, à auto eficácia, ao enfrentamento, à motivação, à fissura e aos estados emocionais. Sendo assim, os determinantes intrapessoais podem ser entendidos como inerentes ao indivíduo, e os determinantes interpessoais inerentes ao contexto no qual o indivíduo está inserido. Faz-se necessário, ainda, ressaltar que tanto os determinantes interpessoais quanto os intrapessoais, por vezes, associam-se uns com os outros no contexto do processo de recaída de dependentes químicos. Portanto, é necessário entender como esses fatores influenciam nas recaídas no contexto da dependência química e de que forma se pode combater esses determinantes, auxiliando assim no tratamento dos pacientes de maneira multidisciplinar (BURIOLA AA, et al., 2011).

Assim, o estudo de Buriola AA, et al., (2011) observou que a intervenção deve estar dirigida ao contexto social do sujeito, já que as análises sugerem a existência de um período crítico para aderência ao tratamento e ressaltam a importância do acolhimento dos usuários do serviço, sendo necessário, portanto, uma interferência ainda dentro deste período.

Em outro estudo, ao realizar a análise destes períodos, observou-se que a cronicidade do uso de drogas é outro fator agravante para a permanência no estilo de vida tóxico, além das questões socioculturais, econômicas e familiares. Associações estatisticamente significativas foram observadas, também, entre recaída no primeiro mês e tempo de uso de cocaína/crack e tempo de internação em outro estudo, sendo observado que o maior tempo de uso predispõe a um maior risco de recaída (LOPES-ROSA R, et al., 2015).

Além disso, uma curta duração da abstinência de metanfetamina e atenção insuficiente foram fatores de risco considerados significativos para a recaída entre os usuários de metanfetamina encontrados no estudo de Chen Y, et al. (2015).

Outro ponto importante a ser destacado da história do paciente, além do tempo de uso e do tempo de abstinência, é o número de substâncias utilizadas. Uma história de poli consumo de drogas pode predizer o risco de recaída de heroína entre os pacientes nesses programas de reabilitação obrigatória realizadas no estudo de Chen T, et al. (2018). 
Outrossim, as atribulações, problemas e dificuldades foram elencados por quatro estudos como fatores de risco para a recaída (YANG M, et al., 2015; CARVALHO FRM, et al., 2011; BUCHELE F, et al., 2004; SOCCOL $\mathrm{KLS}$, et al., 2019). O estudo de Yang M, et al. (2015) evidenciou que as atribulações durante a tentativa de abstinência possuem o fator agravante principal para a recidiva, sendo elas as condições socioeconômicas de pobreza, a falta de apoio familiar e a falta de suporte social responsáveis por tornar mais difícil permanecer em abstinência. Já o estudo de Carvalho FRM, et al. (2011) trouxe que a dificuldade de lidar com frustrações desperta o desejo pelo uso de substâncias psicoativas.

Outros problemas como o desemprego, a falta de um lugar para morar foi elencada por outro estudo como responsáveis por abalar o dependente em processo de recuperação e, se suas respostas de enfrentamento a essas dificuldades não forem positivas, certamente ele enfrentará o risco de recidiva. O estudo traz essas situações de ordem física, psíquica e social, como influenciadoras diretas tanto no sucesso quanto no fracasso de uma recuperação, já que a resposta de enfrentamento de cada indivíduo a estes fatores é que irá determinar o caminho a ser seguido durante o tratamento (BUCHELE F, et al., 2004).

Um fato interessante sobre o enfretamento de problemas é a visão, por parte do usuário, de que a droga ajuda a esquecer os problemas e que, com o uso da droga, o usuário tem expectativas de sentir alegria e bem-estar. Essa constatação foi elencada no estudo de Soccol KLS, et al. (2019) por mulheres assistidas em um Centro de Atenção Psicossocial Álcool e Drogas.

Além disso, o estudo de Álvarez TB e Morales SJ (2015) constatou que as mulheres percebem os estados emocionais negativos e vivenciam emoções positivas como situações de maior risco de recaída do que os homens, sendo elas mais propensas a sofrerem recaídas, após episódios de atribulações emocionais, que os homens.

Em estudo com usuários de crack observou-se que os sonhos com o uso do crack obrigam o indivíduo, de forma involuntária, a passar pela experiência do uso novamente, ou seja, o consumo, os ambientes, as pessoas, as sensações e o prazer. Além disso, a interpretação que os usuários dão aos sonhos parece ser mais importante do que o próprio conteúdo do sonho, o que pode ser usado a favor da terapêutica do paciente (DA SILVA TR e NAPPO AS, 2019).

Observou-se que a família e os amigos podem exercer influências positivas ou negativas durante 0 tratamento do paciente (DA SILVA ML, et al., 2014). Um estudo determinou que o apoio social formal pode diminuir o crime e os comportamentos delinquentes. No entanto, descobriu-se que o apoio formal em consultório, bem como as instalações de tratamento não funcionam com a eficiência esperada em um dos estudos. Dessa forma, cabe aos profissionais de saúde instruir a família, de modo a orientá-los sobre a forma de apoio (XIONG H e JIA J, 2019).

Em outro estudo o uso de exercícios de tipo militar, cronograma apertado, não garantiram a manutenção da abstinência após liberação. Ou seja, a adoção de medidas terapêuticas rígidas, sem muita flexibilidade, ou mesmo a alta carga de cobrança, não trouxeram benefícios para o tratamento dos usuários. A coação para participação dos programas de tratamento resulta, geralmente, em uma baixa motivação para alcançar a abstinência, vendo o tratamento como uma punição (LIL L e HSIAO SC, 2018). Sendo assim, o ideal para uma baixa na recidiva das drogas, é o tratamento em conjunto da sociedade e da família, dando um suporte emocional, mental, social e familiar aos usuários (LIL L e HSIAO SC, 2018; BUCHELE F, et al., 2004).

Dessa forma, nota-se que o tratamento da dependência química é bastante complexo, visto que é necessário um conhecimento interdisciplinar para combater os sintomas físicos, psíquicos e sociais da dependência química. É necessária uma ampla rede de apoio aos profissionais de saúde para tratar da doença.

Assim, percebe-se que para se manter limpo, sem recaídas, um combate é travado pelo dependente contra os estímulos sociais e as reações fisiológicas do organismo. Esse processo depende de fatores externos e internos (determinantes) que envolvem o reconhecimento do padrão de consumo, a classe da dependência, a teia de apoio social, e as estratégias de enfrentamento vinculada ao tratamento (BURIOLA AA, et al., 2011). 
O apoio familiar no tratamento foi um dos aspectos relatados pelos adolescentes em um estudo. A ausência desse apoio foi relacionada com o abandono do tratamento, o que também é descrito pela literatura. Outro fator relatado foi o acesso à droga, comuns em festas e no cotidiano, e, por fim, referiram não adequações do tratamento aos pacientes (como uma equipe que não acolhe e não forma um vínculo, atividades propostas pelo tratamento que não sejam interessantes aos adolescentes, a localização de difícil acesso do serviço especializado ou o horário de funcionamento desfavoráveis à demanda desses adolescentes). Os aspectos identificados neste estudo, colaboram com os achados da literatura sobre os fatores que dificultam o tratamento (VASTERS GP e PILLON SC, 2011).

\section{CONSIDERAÇÕES FINAIS}

Os principais fatores responsáveis pela recaída de dependentes químicos no uso de drogas podem ser subdivididos dentro de determinantes interpessoais e intrapessoais. Fatores como longo período de abuso de substâncias, bem como o curto período de abstinência e o poli consumo, podem ser elencados como fatores importantes para a recidiva. Outro fator observado é a recorrência de problemas e atribulações na vida do dependente, que, frequentemente, o leva ao episódio de recaída. A falta de apoio social, e uma terapêutica militar, também foram elencados como situações desfavoráveis a permanência do paciente em abstinência. Assim, situações de ordem física, psíquica, emocional e social foram elencadas como importantes influenciadoras tanto no sucesso quanto no fracasso de uma recuperação. Sendo, o ideal, um tratamento em conjunto da sociedade e da família, através de um suporte multidisciplinar aos usuários, de maneira longitudinal, por profissionais de saúde que busquem constantemente aprimorar seus conhecimentos acerca da temática, a fim de estarem capacitados para prestar um cuidado integral a esses pacientes.

\section{REFERÊNCIAS}

1. ALMEIDA RBF. O tratamento da dependência na perspectiva das pessoas que fazem uso de crack. Interface Comunicação, Saúde, Educação. 2018; 22: 745-756.

2. ÁLVAREZ AMA. Fatores de risco que favorecem a recaída no alcoolismo. Jornal Brasileiro de Psiquiatria. 2007: 56(3): 188-193.

3. ÁLVAREZ TB, MORALES SJ. Factores de riesgo que influyen en la recaída de consumo de drogas lícitas e ilícitas en adolescentes atendidos en el Instituto sobre Alcoholismo y Farmacodependencia. Revista Costarricense de Psicología. 2015; 34(2): $147-157$.

4. BATOOL S, et al. Pattern of addiction and its relapse among habitual drug abusers in Lahore, Pakistan. Eastern Mediterranean Health Journal La Revue de Santé de la Méditerranée orientale. 2017; 23(3).

5. BÜCHELE F, et al. Dependência química e prevenção à "recaída". Texto \& Contexto Enfermagem. 2004; 13(2): 233-240.

6. BURIOLA AA, et al. Análise de determinantes intrapessoais e interpessoais como motivos de recaída no contexto da dependência química. Journal nursing and health. 2018; 8(2).

7. CARVALHO FRM, et al. Causas de recaída e de busca por tratamento referidas por dependentes químicos em uma unidade de reabilitação. Colombia Médica. 2011; 42(2): 57-62.

8. CHAN GHY, et al. Intrinsic Motivation and Psychological Connectedness to Drug Abuse and Rehabilitation: The Perspective of SelfDetermination. International Journal of Environmental Research And Public Health. 2019; 16(11): 1934-1951.

9. CHEN T, et al. Polydrug use patterns and their impact on relapse among heroin-dependent patients in Shanghai, China. Addiction. 2018; 114(2): 259-267.

10. CHEN Y, et al. Predictors of Relapse and Dropout During a 12-Week Relapse Prevention Program for Methamphetamine Users. Journal of Psychoactive Drugs. 2015; 47(4): 317-324.

11. DA SILVA ML, et al. Fatores de risco e proteção à recaída na percepção de usuários de substâncias psicoativas. Revista da Rede de Enfermagem do Nordeste. 2014; 15(6).

12. DA SILVA TR, NAPPO SA. Crack e sonhos: a visão dos usuários. Ciência \& Saúde Coletiva. 2019; 24(3): 1091-1099.

13. FARRELL MR, et al. Modeling cocaine relapse in rodents: Behavioral considerations and circuit mechanisms. Progress in NeuroPsychopharmacology and Biological Psychiatry. 2018; 87: 33-47.

14. FERNANDES SS, et al. Evasão do tratamento da dependência de drogas: prevalência e fatores associados identificados a partir de um trabalho de Busca Ativa. Cadernos Saúde Coletiva. 2017; 25(2): 131-137.

15. LIU L, HSIAO SC. Chinese Female Drug Users' Experiences and Attitudes With Institutional Drug Treatment. International Journal Of Offender Therapy And Comparative Criminology. 2018; 62(13): 4221-4235.

16. LOPES-ROSA R, et al. Predictors of early relapse among adolescent crack users. Journal Of Addictive Diseases. 2015; 36(2): 136143.

17. LU L, et al. Traditional Medicine in the Treatment of Drug Addiction. The American Journal of Drug and Alcohol Abuse. 2009; 35: 111.

18. OVEISI S, et al. The impact of motivational interviewing on relapse to substance use among women in Iran: a randomized clinical trial. Bmc Psychiatry. 2020; 20(1).

19. RIGOTTO SD, GOMES WB. Contextos de abstinência e de recaída na recuperação da dependência química. Psicologia: Teoria e pesquisa. 2002; 18(1): 95-106.

20. SOCCOL KLS, et al. Motivos da recaída ao uso de drogas por mulheres na perspectiva da fenomenologia social. Enfermagem em Foco. 2019; 10(4): 117-122.

21. VASTERS GP, PILLON SC. O uso de drogas por adolescentes e suas percepções sobre adesão e abandono de tratamento especializado. Revista Latino-Americana de Enfermagem. 2011; 19(2): 317-324.

22. XIONG H, JIA J. Situational Social Support and Relapse: An Exploration of Compulsory Drug Abuse Treatment Effect in China. International journal of offender therapy and comparative criminology. 2019; 63(8): 1202-1219.

23. YANG M, et al. From Abstinence to Relapse: A Preliminary Qualitative Study of Drug Users in a Compulsory Drug Rehabilitation Center in Changsha, China. Plos One. 2015; 10(6). 